\title{
Percepción gerencial del sistema de información empresarial en la problemática ambiental de Ecuador
}

\author{
Mauricio QUISIMALIN \\ Universidad Técnica de Ambato (Ecuador) \\ Javier De EsTeban CURIEL \\ Universidad Rey Juan Carlos (España) \\ javier.deesteban@urjc.es \\ Arta ANTONOVICA \\ Universidad Rey Juan Carlos (España) \\ arta.antonovica@urjc.es
}

Recibido: 24 de junio de 2014

Enviado a evaluar: 4 de julio de 2014

Aceptado: 10 de septiembre de 2014

\section{RESUMEN}

Este estudio analiza desde el punto de vista gerencial y ambiental, el sistema de información empresarial en la problemática de gestión de desechos en papel, plástico y pilas dentro del contexto comunitario de la Provincia de Tungurahua- Ecuador, en donde se utilizó un procedimiento estadístico descriptivo en la investigación. Se indagó a 385 empresas de Tungurahua de la base de datos de la "Cámara de Comercio de Ambato", encuestando a sus responsables relacionados con temas ambientales. Entre los aspectos relevantes del estudio, se ponen de manifiesto: a) el $41 \%$ de las empresas encuestadas percibe que los elementos que administra un sistema de información empresarial son identificar procesos, métodos y herramientas en la gestión de residuos para satisfacer las necesidades de los consumidores, lo que indica que la mayoría no identifica una definición de lo que es un sistema de información; b) por otro lado, se percibe la falta de concienciación ambiental, ya que solo el $20 \%$ de las empresas de Tungurahua indican que debe existir una mayor responsabilidad con respecto a la gestión ambiental.

Palabras clave: Sistema de Información Empresarial, Percepción Gerencial, Gestión de Desechos Sólidos, Papel, Plástico, Pilas. 


\title{
Managerial perception of business information system in the environmental issue of Ecuador
}

\begin{abstract}
This paper analyzes from the management and environmental point of view, the business information system in the issue of paper, plastic and batteries waste management within the community context of the province of Tungurahua, Ecuador, where a descriptive statistical method was used in this study. The research was conducted on 385 companies Tungurahua database of the "Chamber of Commerce of Ambato," surveying its related environmental issues responsible. Among the relevant aspects of the study, it must be highlighted: a) $41 \%$ of the companies perceived that the elements managed by a business information system are to identify processes, methods and tools in waste management to meet the needs of consumers, indicating that the majority does not identify a definition of what is an information system; b) on the other hand, the lack of environmental awareness is perceived as only $20 \%$ of Tungurahuan companies indicate that there must be a greater responsibility for environmental management.
\end{abstract}

Key words: Business Information System, Managerial Perception, Solid Waste Management, Paper, Plastic, Batteries.

\section{Perception des gérants sur le système d'information de l'entreprise et les problèmes environnementaux de l'Equateur}

\section{RÉSUMÉ}

Cette étude analyse du point de vue des gérants et de l'environnement, le système d'information d'entreprise dans le problème de la gestion des déchets en papier, en plastique et des batteries dans le cadre de la communauté de la province de Tungurahua, Equateur, où une méthode statistique descriptive a été utilisée dans la recherche. Cette recherche a été conduite sur 385 entreprises de la base de données de Tungurahua "Chambre de Commerce d'Ambato", avec une enquête de questions environnementales aux responsables de ces entreprises. Parmi les aspects pertinents de l'étude, on doit mentionner: a) 41\% des entreprises interrogées, percoivent le système d'information d'entreprise avec les processus, méthodes et outils de gestion des déchets pour répondre aux besoins des consommateurs, indiquant que la majorité ne permet pas d'identifier une définition de ce qu'est un système d'information; b) d'autre part, la manque de sensibilisation à l'environnement est perçu car seulement $20 \%$ des entreprises indiquent qu'en Tungurahua doit y avoir une plus grande responsabilité de la géstion de l'environnement.

Mots clé: Système d'information de l'entreprise, Perception des gérants, Gestion des déchets solides, Papier, Plastique, Piles.

\section{INTRODUCCIÓN}

La sociedad ecuatoriana tiene una preocupación en la mejora del medio ambiente ha inducido en el espacio empresarial, nuevos retos que causan la introducción de las variables medioambientales.

Actualmente se discute los actuales sistemas de producción que contraen el ciclo de vida del producto para integrar una demanda en aumento, debiendo optimizar la relación de la empresa con su entorno. Esto supone un aumento en el deterioro de 
este último, dado que la empresa urge de un entorno que le suministre de la mayor cantidad de recursos posibles y que sea competente de absorber todo los desechos que genere.

La Comisión Mundial del Medio Ambiente y Desarrollo de las Naciones Unidas (Comisión Brutland) ${ }^{1}$ acuñó el término "desarrollo sostenible", que se refiere al crecimiento económico con el cuidado del medio ambiente, lo cual parecía antagónico hasta el momento. El desarrollo sostenible de Ecuador no implica la adopción de un modelo económico que se pretenda recuperar el patrimonio ambiental a sus niveles originarios, sino la instauración de políticas de apoyo estructural que compatibilicen los objetivos de crecimiento económico con la conservación del medioambiente.

La protección ambiental por parte de las empresas del Ecuador presenta como principal dificultad el conocimiento de las obligaciones legales. Esta dificultad se deriva en cuanto al conocimiento de las normativas ambientales en los últimos años del Gobierno de Rafael Correa, obligando a las empresas a esforzarse por mantener una continua actualización, y por otro lado la dificultad ante la complejidad técnica de estas normas. Los conflictos de índole social, ambiental y económico que promueven a desarrollarse, en ocasiones incluso con virulencia, aconsejan poner freno a la tendencia actual porque no aborda los retos principales de nuestra sociedad, es decir, no aborda las variables relacionadas con la sostenibilidad y las que indican que entramos en una nueva era: la era de la información y el conocimiento.

La ciudad de Ambato capital de la Provincia de Tungurahua - Ecuador, razonablemente compacta y compleja se revela como un modelo de ciudad más sostenible. Por ello, con los cambios y mejoras necesarias, se deberá adaptar a los criterios de la sostenibilidad y también a los criterios que hagan de nuestros sistemas urbanos, modelos de ciudad del conocimiento. Trabajar de manera integrada por un modelo de ciudad sostenible y a la vez del conocimiento parece la mejor estrategia para competir.

Ante este planteamiento inicial, ¿por qué un sistema de información en la problemática de la gestión de desechos sólidos en papel, plástico y pilas dentro del contexto comunitario de la Provincia de Tungurahua - Ecuador? La respuesta no es fácil de responder, ya que se necesita de posteriores investigaciones que se vincule con este estudio, si bien en esta comunicación se pretende modestamente responder con criterios científicos.

${ }^{1}$ Informe Brundtland. Es un informe que enfrenta y contrasta la postura de desarrollo económico actual junto con el de sostenibilidad ambiental, realizado por la ex-primera ministra de Noruega Gro Harlem Brundtland, con el fin de analizar, criticar y reformular las políticas de desarrollo económico en la globalización, reconociendo que el actual avance social se está llevando a cabo a un costo medioambiental alto. Implica un cambio muy importante en cuanto a la idea de sustentabilidad, principalmente ecológica, y a un marco que da también énfasis al contexto económico y social del desarrollo. 
De este modo, la implantación de un sistema de información empresarial no sólo genera un beneficio social, sino que también puede generar suculentos beneficios a las empresas privadas y públicas, a través de la consecución de mejoras de la competitividad empresarial, propiciadas fundamentalmente, por aumentos de demanda, incrementos de productividad, reducción de costos, alimentación de la información, entre los principales beneficios.

Así mismo, la adecuada gestión de los aspectos medio ambientales tiene un costo que para algunas empresas llega a ser elevado, siendo la mejor forma de reducir estos costos la minimización de la contaminación generada, y aprovechando las ventajas comerciales y de imagen que ofrece la protección ambiental.

Por todo ello, la gestión medioambiental debe cambiar de enfoque, abandonando la línea tradicional en la que se introducen medidas técnicas de corrección al final de la cadena de producción, incorporando las nuevas técnicas de gestión empresarial basadas en el seguimiento y control de todas las fases del proceso productivo mediante la información: en otros palabras, sistemas de información empresarial sobre cuestiones ambientales.

\section{EL SISTEMA DE INFORMACIÓN Y LA GESTIÓN DE DESECHOS SÓLIDOS EN PAPEL, PLÁSTICO Y PILAS}

El sistema de información empresarial ayuda a los negocios a ser más flexibles y productivos, al coordinar sus procesos de negocios más de cerca e integrar grupos de procesos, de modo que se enfoquen en la administración eficiente de los recursos y el servicio al cliente (Laudon y Loudon, 2012: 51) El sistema de información implica básicamente aspectos económicos a través del establecimiento de políticas empresariales y de control que permiten comparar los resultados obtenidos con los objetivos perseguidos, así como con otros estándares del sector. De este modo, se depuran responsabilidades y se corrigen posibles desviaciones que afecten a la empresa y al medio ambiente en lo que se refiere a los desechos de papel, plástico y pilas.

"Los Sistemas de Información existen en todo tipo de organizaciones y son fundamentales para que éstas puedan obtener, procesar, almacenar y gestionar su información. Se puede decir entonces, que el propósito de los Sistemas de Información es proveer datos e información a quien la necesite dentro de la organización a quien sirve. Asimismo, un Sistema de Información se puede ver como un sistema de procesamiento de datos que actúa sobre los datos puros para producir Productos de Información" (Caro; Fuentes y Soto, 2013: página web).

Por otro lado, "los sistemas de información en la organización, no son algo nuevo. Desde mucho antes de utilizar los computadores para la automatización, ya las organizaciones reunían, almacenaban y actualizaban información de sus actividades diariamente manualmente. En el pasado como en el presente, los sistemas de información consisten en estándares establecidos para el procesamiento, almacenamiento y entrega de información a los miembros de la organización donde cada una de estas personas, requiere información distinta para la realización de su 
trabajo, en particular como instrumento en la toma de decisiones y en las actividades de control gerencial" (Bencomo, 2012: página web).

Además, "una de las principales funciones que debe realizar el sistema de información de una organización es proporcionar la información necesaria a la alta dirección para la adecuada toma de decisiones. Esta información se resume en los resultados de la contabilidad de la empresa, entendidos en sentido amplio, es decir, no sólo el resultado del ejercicio, sino la información contenida en el balance, la cuenta de pérdidas y ganancias y demás estados económicos y financieros que se hayan establecido como relevantes en la organización concreta". (Solana Álvarez, 2014:473).

Entrelazando los sistemas de información y el medio ambiente, se puede decir que el medio ambiente debe proporcionar a la empresa funciones diversas que son de gran trascendencia para la misma, como: 1) la provisión de una fuente fundamental de recursos naturales; 2) la concepción de la naturaleza como un nuevo mercado de bienes y servicios relacionado con el ocio y disfrute del medio ambiente; 3) la absorción de los residuos, emisiones y vertidos que generan las empresas como consecuencia de su proceso productivo.

Bajo este contexto, la "Cámara de Comercio de Ambato" ${ }^{2}$ - Tungurahua ha brindado su base de datos para la elaboración de esta investigación, ya que no había estudios relacionados con este tema ambiental. Si bien esta organización lleva años dedicados a informar a las empresas sobre asuntos empresariales, no existía una investigación ambiental en este sentido. Esta indagación facilita el inicio de futuras investigaciones más profundas, con el objeto de facilitar el conocimiento y promover la reducción en origen de la contaminación, eficiencia y el ahorro energético como la forma de gestión más competitiva y ambientalmente razonable. Además, existen pocos trabajos académicos en Ecuador sobre sistemas de información empresarial y gestión de desechos sólidos (ninguna investigación en Tungurahua) sobre la aplicación, adaptación, compresión e implicación sobre este tema de estudio en particular.

El autor O'Brien (2007: 4) sostiene que "un sistema es un conjunto de componentes interrelacionados con un límite claramente definido, que trabajan juntos para lograr una serie de objetivos en común". Por su parte, De Pablos Heredero et al (2006: 558) manifiestan que el desarrollo de un sistema de información es un proyecto que, independientemente de su tamaño, es complejo. A menudo requiere cambios en la organización: ingeniería de software, el desarrollo de aplicaciones y por extensión de sistemas pasó de ser una disciplina artesanal e implícita, a ser un verdadero trabajo de ingeniería, de construcción, técnico y explicito, mejor evaluable y controlable.

La minimización de desechos es una de las propuestas que realiza las tendencias de la ecoeficiencia. El establecimiento de un programa de minimización de desechos

${ }^{2}$ La Cámara de Comercio de Ambato (2012) brindó base de datos actualizados para la realización de esta información 
es análogo a un programa total de la calidad. En el caso del programa de minimización de desechos, aun las compañías más eficientes producen desechos, el costo real de estos desechos se encuentra alrededor del $4 \%$ del volumen del negocio. Sin embargo, con unas herramientas adecuadas, esta cifra se puede reducir en una cuarta parte, con un ahorro significativo para la empresa, dependiendo de la eficiencia de la misma en el uso de sus recursos en general.

Los sistemas de gestión ambiental son implementados por compañías que quieren mejorar la gestión del desempeño ambiental a través del tiempo. Estos sistemas (véase Figura 1) cumplen con las responsabilidades y tareas de todas las personas en la organización. A través de un sistema totalmente integrado, entendible, comprensible y abierto, la degradación ambiental puede evitarse según los objetivos del milenio del Programa de las Naciones Unidas ${ }^{3}$

Figura 1. Estructura del sistema de información empresarial

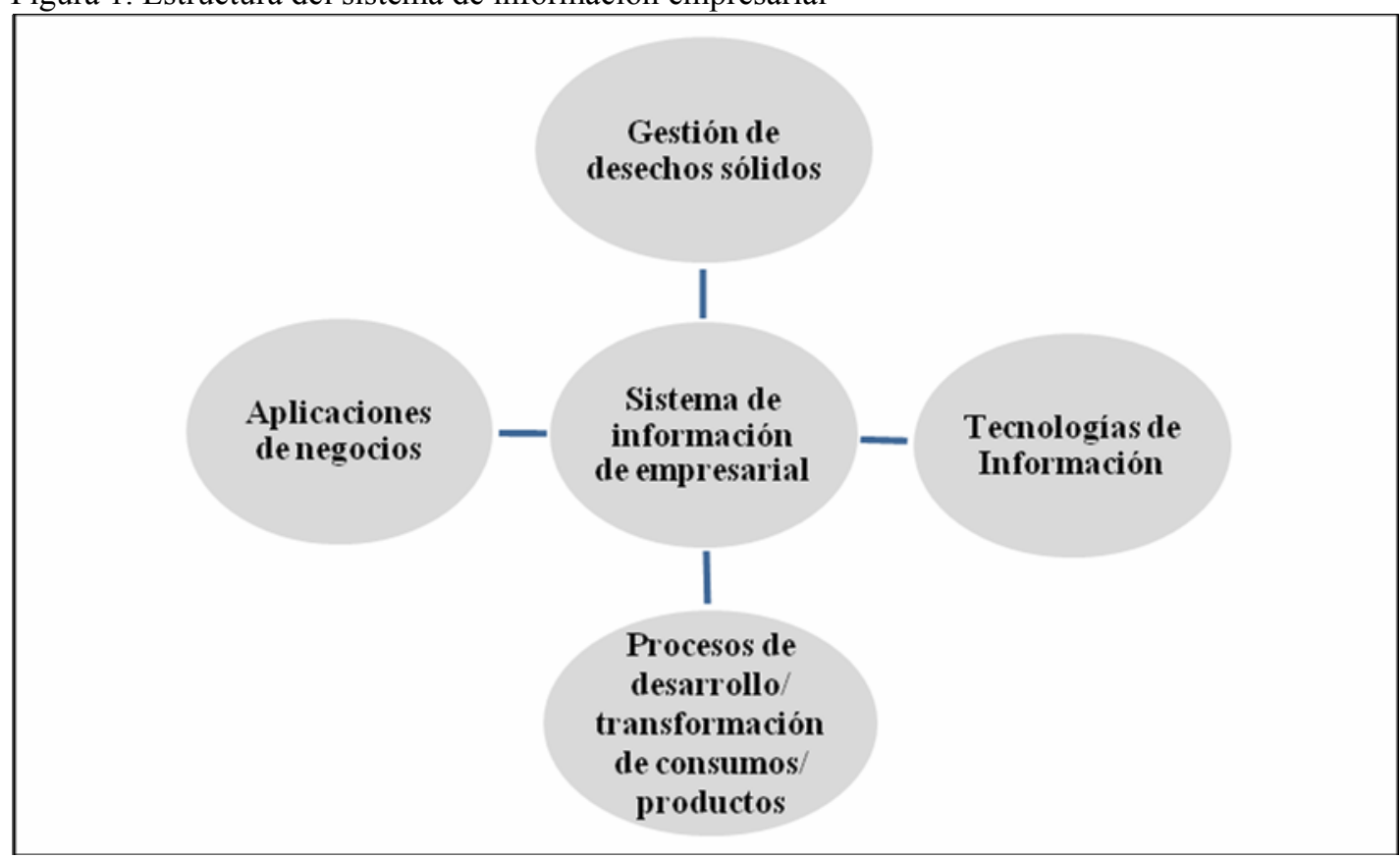

Fuente: Adaptado de la Cámara de Comercio de Ambato (2012, nota de página nº2, comentada anteriormente)

${ }^{3}$ Objetivo 7: Garantizar el sustento del medio ambiente (objetivos del milenio PNUD) 
Una gestión adecuada de los residuos sólidos tiene como objetivo la optimización de las inversiones y de los costes operacionales necesarios para reducir la cantidad de los residuos y el impacto ambiental que puedan producir hasta límites que se consideran aceptado de acuerdo con los conocimientos actuales.

El científico Bertalanffy (1989: 356) sintetiza que se debe comprender la organización en su complejidad total (un sistema), en lugar de considerarla simplemente a través de un componente o un área funcional. El estudio de un sistema de producción no produce un análisis satisfactorio si se deja de lado el sistema de comercialización por ejemplo, y por ende la "Teoría General De Sistemas". Por ello, esta aseveración sería aplicable a cualquier empresa en donde se busca gestionar de forma más eficiente sus desechos, por lo que un sistema de información general parece ser la solución más apropiada al respecto.

\section{LA APLICACIÓN DEL SISTEMA DE INFORMACIÓN EMPRESARIAL PARA LA GESTIÓN DE DESECHOS SÓLIDOS}

El mismo autor Bertanlaffy (1987: 323) manifiesta que un sistema, desde el punto de vista matemático, es una operación de adición que puede representarse como una relación ternaría, esto es: un conjunto ordenado de tres elementos que satisface dicha relación entre sí, y solamente si se suman los dos primeros elementos, obtenemos el tercer elemento, como por ejemplo:

$$
\mathrm{R}(\mathrm{a}, \mathrm{b}, \mathrm{c}) \leftrightarrow \mathrm{a}+\mathrm{b}=\mathrm{c}
$$

En consecuencia, la Teoría General de Sistemas, al igual que todas las ciencias verdaderas, se basa en una búsqueda sistemática de la ley y el orden en el universo; pero a diferencia de las otras ciencias, tiende a ampliar su búsqueda, convirtiéndola en una búsqueda de un orden de órdenes, de una ley de leyes. Este es el motivo por el cual se le ha denominado la Teoría General de Sistemas.

En la siguiente Figura 2, se observa como se propone un modelo de sistema de información empresarial para la aplicación de desechos sólidos en papel, plástico y pilas en las empresas de Tungurahua (Ecuador), y donde están interconectados tres subsistemas. El primero (subsistema uno) del lado izquierdo es: los participantes del entorno como son la comunidad, los gobiernos locales, las universidades, los colegios, escuelas, municipios, las empresas, y los clientes. En este subsistema, se encuentran los recursos económicos, los recursos materiales que realizan el proceso de negocios/ servicios o transformaciones de productos que luego se transforman en productos finales o servicios y que van al consumidor final.

El subsistema dos está conformado por un sistema de información, donde se maneja la comunicación, los insumos que entran y salen (sean estos en forma de productos terminados o como desechos). Estos insumos pasan luego a la gestión de desechos sólidos como subsistema tres, en donde la generación, transporte, almacenamiento, recolección, tratamiento y disposición final, se administra por las 
políticas públicas de medio ambiente de Ecuador, la información ciudadana, la construcción de indicadores a partir de la demanda de los usuarios de información, los registros administrativos ambientales, las encuestas y los censos que ayudarán a realizar estimaciones medioambientales en la Provincia de Tungurahua.

Figura 2. Sistema de información empresarial en la gestión de desechos sólidos

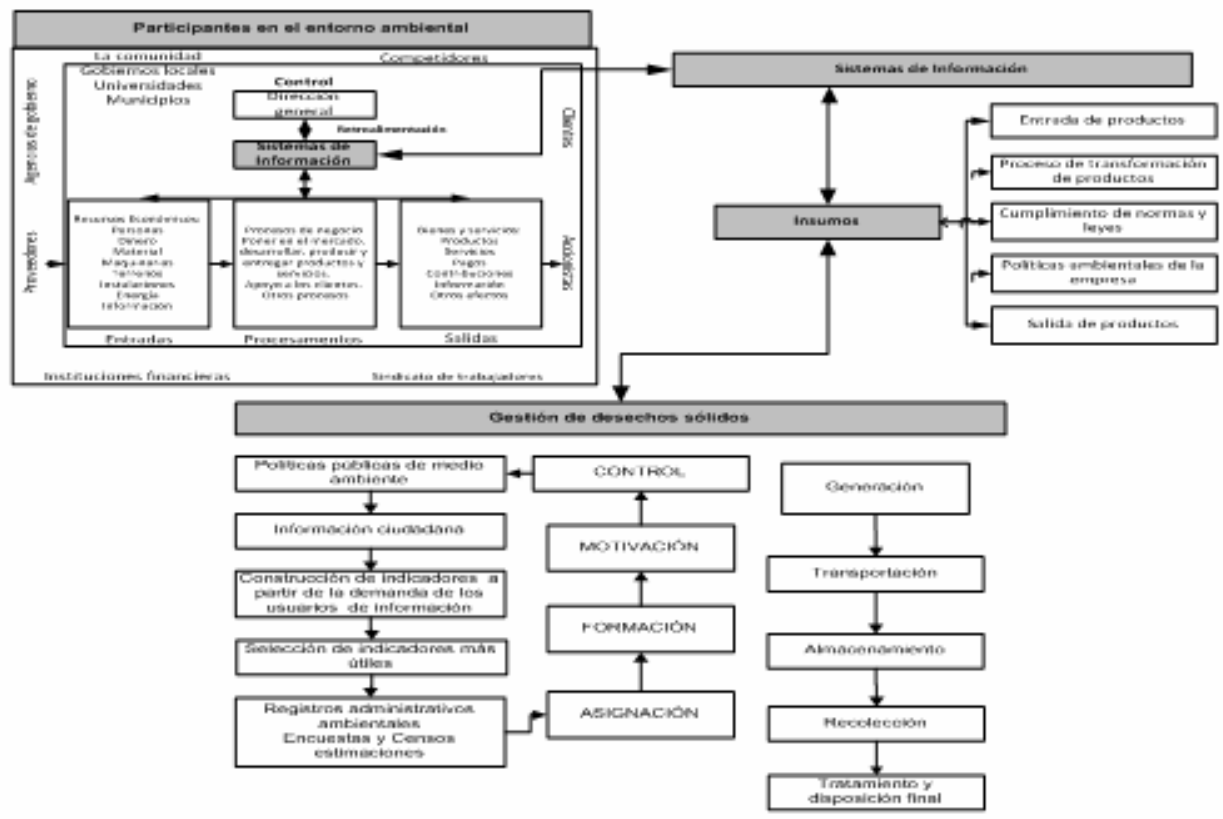

Fuente: Adaptado de la Cámara de Comercio de Ambato (2012, nota de página $\mathrm{n}^{\circ} 2$ comentada anteriormente)

\section{METODOLOGÍA}

El investigador Rodríguez Moguel (2005: 1) define que la investigación "es un procedimiento reflexivo, sistemático, controlado y crítico, que permite descubrir nuevos hechos o datos, relaciones o leyes, en cualquier campo del conocimiento humano." También los investigadores Veal (1997) y Brunt (1998) sostienen que los resultados de la investigación ayudan a comprender, aclarar, pronosticas fijar políticas, planificar y tomar decisiones de gestión. De este modo, esta investigación se define a partir del enfoque cualitativo y cuantitativo para la toma de decisiones, implementando similitudes y diferencias de ambos enfoques. En consecuencia, se identifican las características esenciales de cada enfoque, y se destaca que ambos son herramientas igualmente valiosas para el desarrollo de la ciencia. 
Cuando se elaboró el marco teórico en esta investigación, se efectuó la revisión de literatura especializada en economía ambiental, economía ecológica, sistemas de información empresarial y manejo de desechos sólidos, especialmente en papel, plástico y pilas. Esto ayudó conceptualmente en el diseño de los instrumentos de medición utilizados. Se realizaron primeramente unas entrevistas para la obtención de contenidos y que ayudaron a la elaboración final del cuestionario. En concreto se implementaron 8 entrevistas a personas expertas (en sistemas de información por un lado, y en temas medioambientales por otro), sintetizando la información obtenida en cuadros comparativos. Posteriormente, como fase final se diseñaron los cuestionarios para la encuesta aplicada a los responsables de empresas de la región de Tungurahua (Ecuador). En particular, la muestra (385 empresas, encuestando a sus responsables en temas de gestión ambiental) se obtuvo de la base de datos de socios de la "Cámara de Comercio de Ambato", (1600 empresas en Tungurahua, la mayoría asentadas en el cantón Ambato, capital de Tungurahua). Esta encuesta fue realizada en los 9 cantones $^{4}$ de la región de Tungurahua, siguiendo un muestreo aleatorio simple, y ejecutada con la colaboración de estudiantes universitarios de la Universidad Técnica de Ambato capacitados para este propósito. La validez total del instrumento de medición (cuestionario) se evaluó sobre la base de todos los tipos de evidencia habituales (Alpha de Cronbach, especialmente. En nuestro caso 0.82).

Los científicos Hernández et al (2010: 204) manifiestan que cuanto mayor evidencia de la validez de contenido, de validez de criterio y de validez de constructo se tenga en un instrumento de medición, éste se acercará más a representar la(s) variable(s) que se pretende medir. La objetividad se reforzó mediante la estandarización en la aplicación del instrumento (las mismas instrucciones y condiciones para todos los encuestados participantes) y en la evaluación de los resultados (mismos análisis estadísticos).

De manera resumida, el procedimiento que se siguió para construir el instrumento de medición (cuestionario) fue el siguiente:

1) Construcción del instrumento (pre-cuestionario): esta etapa implicó la generación de todos los ítems y categorías del instrumento a partir de las entrevistas exploratorias previas, así como la determinación de los niveles de medición y codificación de los ítems.

2) Diseño del instrumento de medición ya elaborado, se adaptó y se desarrolló las versiones finales (cuestionario final).

3) Entrenamiento de los estudiantes que administraron el instrumento.

${ }^{4}$ Ambato, Pelileo, Cevallos, Patate, Pillaro, Mocha, Tisaleo, Baños, Quero. 
4) Aplicación del instrumento de medición a los encuestados: fue la oportunidad de confrontar el trabajo conceptual y de planificación.

5) Preparación de los datos para el análisis: se recolectó, se codificó y se tabuló los datos para luego insertarlos en una matriz de estudio.

6) Análisis: en esta parte del estudio se realizó la exploración, razonamiento y síntesis de los datos obtenidos.

\section{RESULTADOS}

Los principales resultados se exponen a continuación en base a los 385 encuestados (esto es, las personas de la alta dirección de las 385 empresas seleccionadas y comentadas en el capítulo de Metodología).

La categorización de las empresas en Tungurahua arrojó como resultado que el $51 \%$ pertenece al sector del comercio, entre otros, ferreterias, farmacias, almacenes de ropa, accesorios en general; el 30\% a servicios y hoteles; y en menor proporción el $19 \%$ se encuentra el sector industrial como empresas de cuero-calzado, carrocerías, metal-mecánicas primordialmente.

Además, en esta investigación realizada a 385 empresas, el $41 \%$ de los encuestados percibe que los elementos administrados en un sistema de información empresarial son identificar procesos, métodos y sistemas en la gestión de residuos para satisfacer las necesidades de los consumidores. Asimismo, un $20 \%$ de la muestra indagada, menciona que debe existir mayor concienciación con respecto a la gestión ambiental.

Los factores internos que hacen dificil la gestión de desechos sólidos indican que en el $30 \%$ de las empresas de la muestra no existen programas de reciclaje por parte de las mismas; el 16\% indica que existe una limitada iniciativa por parte de los directivos de la empresa.

Dentro de los factores externos que hacen dificil el manejo de desechos sólidos, el $37 \%$ de los encuestados manifiestan que tienen una percepción de desinterés, indiferencia, incluso apatía. Además en el $29 \%$ de las empresas de la muestra, no existen programas integrales de gestión de desechos sólidos por parte de los municipios locales. Igualmente, el $81 \%$ de la muestra de las empresas indican que no miden los desechos sólidos que producen, sólo un pequeño porcentaje del $19 \%$ miden en $\mathrm{Kg}$ o en metros cúbicos estso desechos.

Según la percepción de los encuestados, se percibe un buen manejo de desechos sólidos en la Provincia de Tungurahua - Ecuador, ya que la mayoría de encuestados (49\%) respondieron (véase Figura 3) que gestionan bien la responsabilidad social y ambiental de los desechos sólidos; seguido del $28 \%$ que gestionan bien la imagen ambiental de los mismos; posteriormente el $20 \%$ opina que tratan correctamente la limpieza de desechos; finalmente un porcentaje pequeño del 3\% dice que no realizan nada específico sobre el manejo de desechos sólidos. 
Figura 3. De los siguientes literales: ¿Cree Ud. que brinda un buen manejo de desechos sólidos a la Provincia? Elija una respuesta por favor (\%).

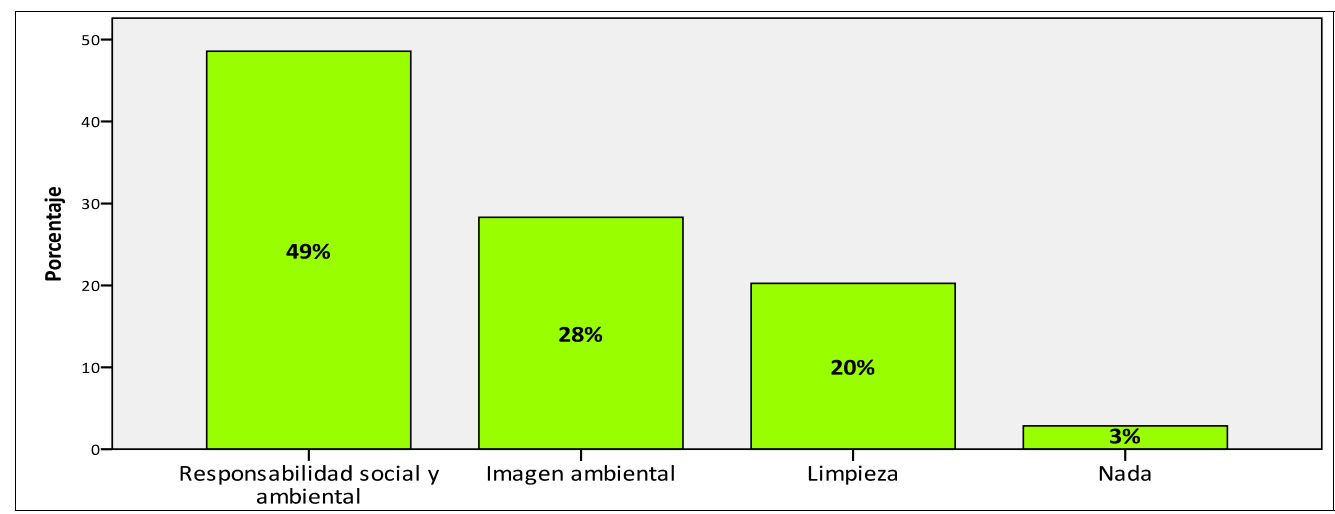

Fuente: Elaboración propia.

En otro orden., la encuesta indica que el $64 \%$ de los gerentes eligen un producto sin fijarse en que sea reciclable (véase Figura 4). En menor medida, el 36\% de los encuestados si optan por adquirir un producto reutilizable, lo que manifiesta una limitada ausencia de conocimiento sobre el reciclaje en productos.

Figura 4. ¿Cuándo eligen un producto, se fija en el hecho que sea reciclable o reutilizable?

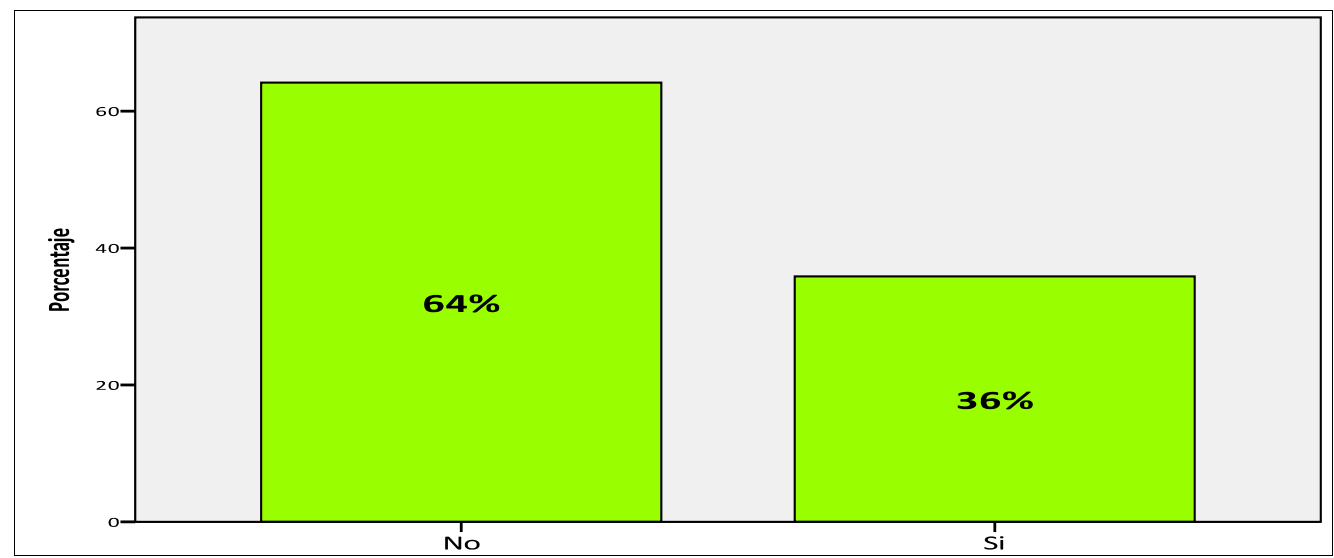

Fuente: Elaboración propia.

Según la opinión de los encuestados, el 42\% (véase Figura 5) piensan que un gerente debería liderar un programa de gestión de desechos sólidos; seguidamente un 
$15 \%$ cree que debería realizarse por los colaboradores de la empresa; el $5 \%$ estima que los clientes deberían liderar parte del programa; y el 3\% sostiene que los proveedores de productos deberían tener la iniciativa en este sentido. Como resultado final, un porcentaje del $36 \%$ considera que deberían participar todos los agentes de una empresa conjuntamente en este programa para la preservación del medio ambiente.

Figura 5. Dentro de su empresa ¿Quién cree Ud. que debería liderar un programa de gestión de desechos sólidos? Elija una respuesta por favor (\%).

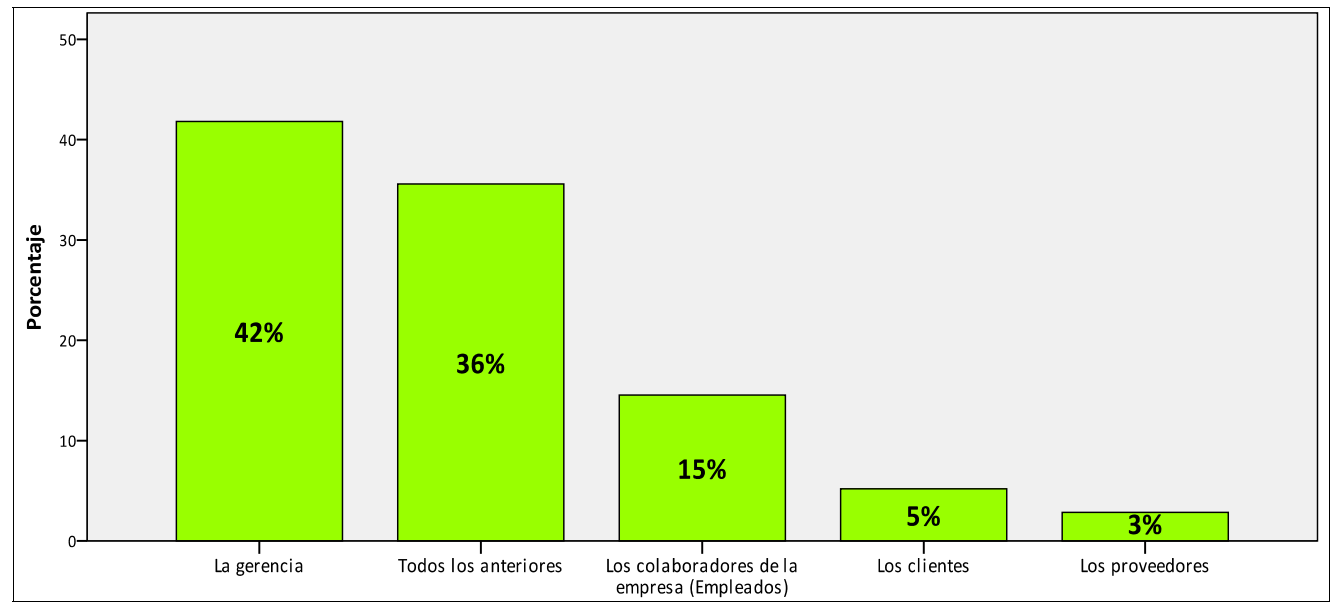

Fuente: Elaboración propia.

Otro de los puntos del cuestionario de esta investigación, analizaba el escenario futurible sobre cómo realizar una campaña de gestión de desechos sólidos y su principal beneficiario. Así, el escenario ambiental fue considerado por un 35\% (véase Figura 6); el $25 \%$ dedicaría la campaña a la imagen empresarial de la organización; posteriormente el $9 \%$ piensa debería concentrarse en las ayudas sociales porque todos saldrían beneficiados. Por otro lugar, el 3\% de la población encuestada piensa que la campaña de gestión de desechos sólidos debería versar sobre los factores económicos. 
Figura 6. Si en su empresa se creara (o existe) una campaña de gestión de desechos sólidos, ¿Qué escenario cree Ud. que sería el beneficiario? Elija una respuesta por favor (\%).

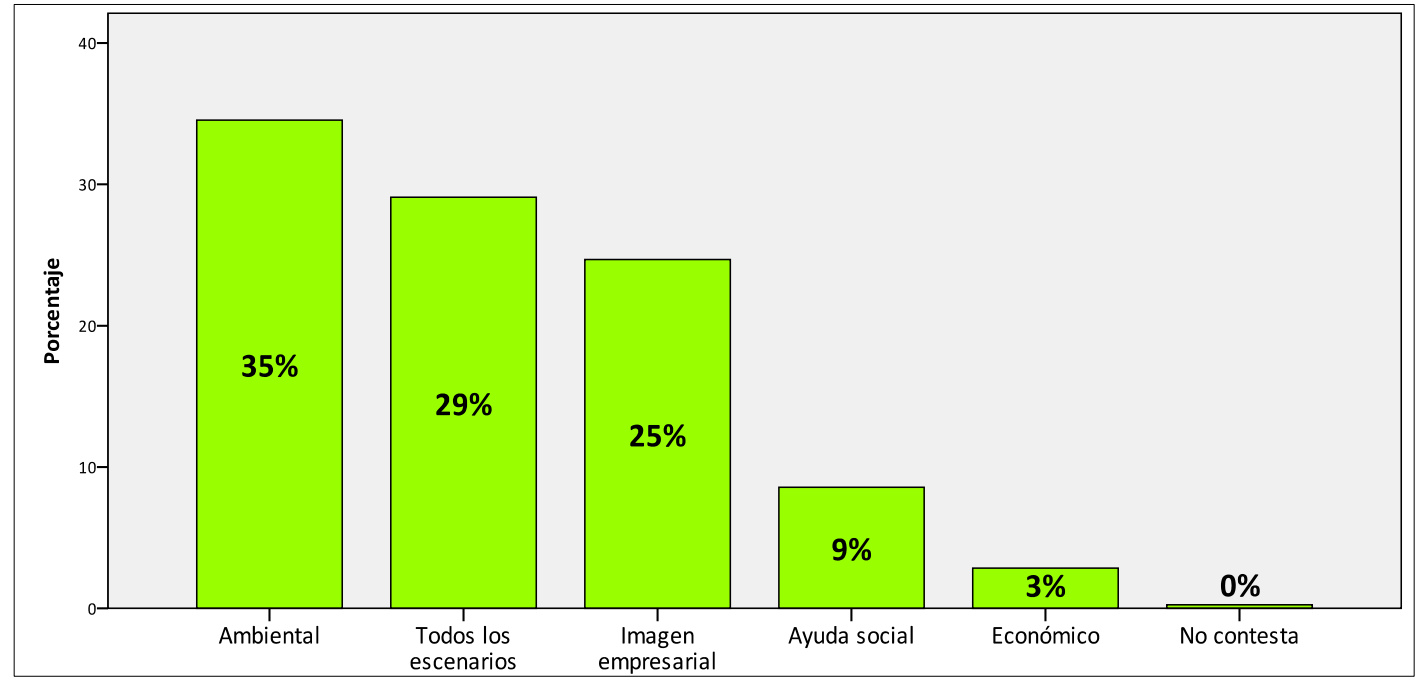

Fuente: Elaboración propia.

Otro pregunta relevante en esta investigación, pretendía averiguar si los gerentes encuestados estarían dispuestos a pagar un precio más alto por un producto que fomente la responsabilidad ambiental. De esta manera, se obtuvo una respuesta positiva en el 68\% de los casos (véase Figura 7); mientras el 30\% se opone a pagar un valor más alto y un $2 \%$ de la muestra no respondió a dicha pregunta.

Figura 7. ¿Estaría dispuesto a pagar un precio más alto por un producto que fomente la responsabilidad ambiental? (\%).

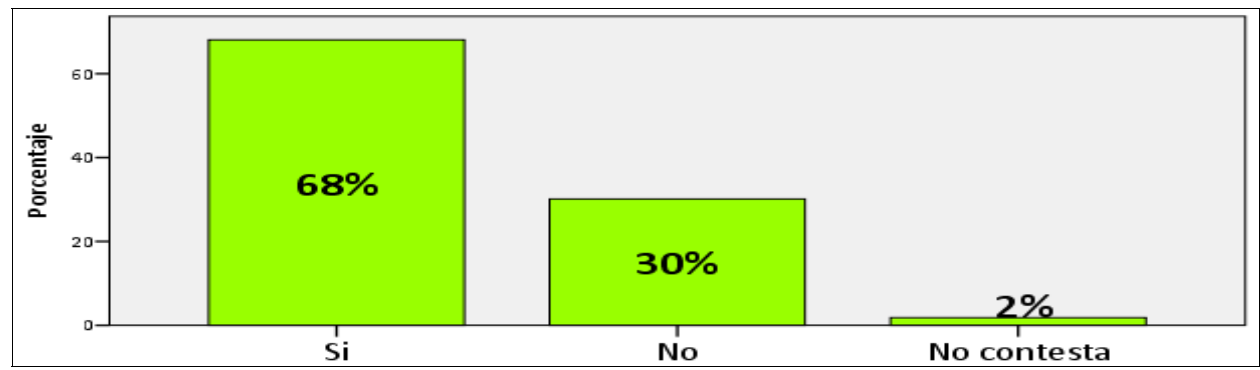

Fuente: Elaboración propia. 
Tabla 1. Tabla comparativa ${ }^{5}$.

\begin{tabular}{|l|c|c|c|}
\hline \multicolumn{3}{|c|}{ Estadísticos } \\
\hline a. Plásticos & Media & Moda & Desv. Std. \\
b. Papel - Cartón & 3.76 & 5 & 1.16 \\
c. Pilas & 3.94 & 4 & 1.00 \\
\hline
\end{tabular}

Fuente: Elaboración propia.

Por último, se puede manifestar que los encuestados dan significativa importancia (véase Tabla 1) a todas las variables relacionadas con la gestión de desechos. En particular, la gestión de desechos de papel- cartón se considera el más "trascendente" (media de 3,94 sobre 5), seguramente debido a que la administración de estos materiales (papel y cartón) es más fácil "reciclar" que los plásticos y las pilas (involucran más tiempo, espacio, tecnología, técnicas y recursos económicos). Asimismo, es substancial precisar que la "desviación estándar" en cada una de las variables ayuda a explicar la distancia que existe con respecto a la media $(\mu)$; la "moda" por otro lado es un indicador que ayuda a aclarar el nivel de frecuencia en la respuesta o calificación en cuanto a la importancia que los entrevistados contestaron en cada una de las variables, estableciendo en todos los valores comparados como "importantes".

\section{CONCLUSIONES Y REFLEXIONES FINALES}

En bases a los resultados de esta investigación, el sistema de información empresarial es importante para la eficiencia operacional, la productividad y la moral de los colaboradores, así como el servicio y satisfacción de los clientes. Las empresas de Tungurahua van progresando en función de los clientes, y por medio de los diferentes gobiernos en el diseño de leyes, normas, regulaciones y políticas públicas, existiendo una mejor eficiencia en la gestión ambiental en papel, plástico y pilas por parte de estas empresas.

Un ingrediente vital a la hora de desarrollar productos y servicios competitivos que provean a una organización de una ventaja estratégica en el mercado global, es tomar en cuenta al medio ambiente y que los desechos sólidos se puedan reducir, reutilizar, o reciclar. Sin embargo, en las empresas de Tungurahua no existen programas generalizados de reciclaje como principales factores internos. Además los factores externos que hacen difícil el manejo de desechos sólidos son el desinterés, la indiferencia, y la apatía por parte de los habitantes de la ciudad.

En este sentido y en base a las evidencias encontradas en este estudio, las empresas no miden los desechos sólidos que producen. Mayoritariamente estas

\footnotetext{
${ }^{5}$ Tabla comparativa entre la importancia que se le asigna a la gestión de desechos de los siguientes materiales en escala del 1 al 5 , donde 1 es nada importante y 5 muy importante (gerentes de las empresas)
} 
empresas eliminan los desechos sólidos diariamente, y muy pocas los reciclan o los reducen, lo que implica la oportunidad de diseñar proyectos o planes de gestión (públicos o privados) de desechos sólidos en papel, plástico y pilas (sobre todo en estas últimas ya que son altamente contaminantes). En consecuencia, los temas de gestión ambiental deberían ser expuestos principalmente en medios de comunicación por parte de los organismos que manejen el tema de administración, brindando no sólo responsabilidad ambiental sino también social. Además, las organizaciones están dispuestas a comprar y vender insumos o productos con materiales retornables y reciclables. Si bien, es importante indicar que las empresas manifiestan que la gerencia debería liderar los programas de gestión de desechos sólidos en papel, plástico y pilas.

Por otro lado, existe un factor principal para no escoger un programa de manejo de desechos sólidos, como son los costos de implementación. Algunos encuestados creen que con un programa de gestión de desechos sólidos, no se puede ahorrar costos dentro de la empresa. Sin embargo, las empresas de Tungurahua estarían dispuestas a contribuir en la producción con un precio más alto pero que fomente la responsabilidad ambiental para la sociedad de Tungurahua. Reciclar, reutilizar, reducir son las estrategias que deben utilizar las empresas para la minimización de residuos sólidos en los materiales antes mencionados. El factor más importante que ayudaría a implementar programas de gestión de desechos sólidos es, sin duda, la motivación de todos los involucrados en el sistema de producción.

Los gerentes manifestaron, igualmente, que el aspecto más importante en la recogida selectiva de desechos sólidos en su cantón es la concienciación ciudadana con respecto a la gestión ambiental, pese a que "no" realizan una gestión de residuos sólidos de plástico, papel o pilas en su hogar. De hecho, los factores que han considerado difíciles para la gestión de desechos sólidos en papel, plásticos y pilas en la ciudad, son la ausencia de basureros especiales para clasificar los desechos por tipo, el desinterés, la indiferencia por parte de los habitantes de la ciudad, y la falta de motivación en gestión de desechos sólidos por parte de municipios locales de la provincia.

Cabe además mencionar que la gran mayoría de las empresas indagadas en esta provincia han manifestado que la separación en origen "sí" contribuye a reducir el problema y que facilita el proceso de recolección, de reutilización, de reducción y/o reciclaje. Además indican que, "no es una pérdida de tiempo", lo que supone una oportunidad para el desarrollo de nuevos productos relacionados en este sentido.

Finalmente, los municipios de las localidades estudiadas "no" aportan suficientes recursos financieros y operativos para la facilitación en origen. Bajo este contexto, las empresas encuestadas indican que todos los involucrados deben realizar la separación en origen, esto es, universidades, colegios, escuelas, empresas, ciudadanía en general deben estar integradas para la realización de un solo esfuerzo. Todo ello, junto con la batuta del Ministerio de Ambiente del Ecuador que según los encuestados de este estudio, debe ser el organismo líder en las políticas públicas de gestión de desechos sólidos en general, y del papel, plástico y pilas en particular. 


\section{BIBLIOGRAFÍA}

BENCOMO F., F. (2012). "Los sistemas de información como atractores en las organizaciones desde una visión compleja". En Observatorio de la Economía Latinoamericana, $\mathrm{N}^{\mathrm{o}}$ 162, Enero 2012. Informacion disponible en HYPERLINK "http://www.eumed.net/cursecon/ecolat/ve/2012/fjbf.html" http://www.eumed.net/cursecon/ecolat/ve/2012/fjbf.html (visitada: 20/05/2014).

BERTALANFFY, L. Von y WEINBERG, G M. (1987) Tendencias en la teoria general de sistemas ( $3^{\mathrm{a}}$ ed.). Madrid : Alianza.

BERTALANFFY, L. Von (1989). Teoria general de los sistemas ( $7^{\mathrm{a}}$ ed.). México: Fondo de Cultura Ecónomica.

BRUNT, P. (1998). Market research in travel and tourism (2nd ed.). Oxford: Butterworth Heinenmann.

CARO, A.; FUENTES, A. y ANTONIETA SOTO, M. (2013). "Desarrollando sistemas de información centrados en la calidad de datos”. En Ingeniare. Revista chilena de ingeniería, vol. $21 \mathrm{~N}^{\circ}$ 1, 2013. Informacion disponible en: HYPERLINK "http://www.scielo.cl/scielo.php?script=sci_arttext\&pid=S071833052013000100006"

http://www.scielo.cl/scielo.php?script=sci_arttext\&pid=S071833052013000100006 (visitada: 18/06/2014).

DE PABLOS HEREDERO, C. y Lopez-Hermoso, J. (2006). Dirección y gestión de los sistemas de información en la empresa. Madrid: ESIC.

HERNÁNDEZ, R. y FERNANDEZ, C. (2010). Metodología de la investigación (5 ed.). México: Mcgraw Hill.

LAUDAN, K. C. y LAUDON, J. P (2012). Sistemas de informacion gerencial. México: Pearson.

O'BRIEN, J. A. (1999). Management Information Systems - managing Information Technology in the internetworked enterprise. Boston: Mc Graw-Hill.

RODRIGUEZ MOGUEL, E. A. (2005). Metodología de la investigación (5a ed.). México: Universidad Juárez Autónoma de Tabasco.

SOLANA ÁLVAREZ, J. M. (2014). "El sistema de información de una organización. Necesidad de implicación de la dirección". En Anuario Jurídico y Económico Escurialense, XLVII. Informacion disponible en HYPERLINK "http://www.rcumariacristina.net:8080/ojs/index.php/AJEE/article/view/202/169" http://www.rcumariacristina.net:8080/ojs/index.php/AJEE/article/view/202/169 (visitada: 02/06/2014).

VEAL, A. (1997). Research methods for leisure and tourism: a practical guide ( $^{\text {nd }}$ ed.). Essex: Pearson Education. 